\title{
Deciphering the genesis and fate of amyloid $\beta$-protein yields novel therapies for Alzheimer disease
}

\author{
Dennis J. Selkoe \\ Center for Neurologic Diseases, Harvard Medical School, Brigham and Women's Hospital, Boston, Massachusetts, USA \\ J. Clin. Invest. 110:1375-1381 (2002). doi:10.1172/JCI200216783.
}

One of the most rewarding moments in science arises when research on seemingly disparate processes begins to reveal a broad, common principle. Such a confluence has occurred recently in the study of several etiologically unrelated brain diseases that display distinct symptoms and pathologies. In a growing number of neurodegenerative disorders, the accumulation of oligomeric assemblies of misfolded proteins is known to induce injury gradually to diverse neuronal populations. Perhaps the foremost example of this phenomenon, both historically and in terms of its societal impact, is Alzheimer disease (AD).

Progressive dysfunction of neurons in the limbic and association cortices underlies the cognitive failure of $\mathrm{AD}$. This cellular dysfunction is preceded by the accumulation of extracellular aggregates of the 42-residue amyloid $\beta$-protein $(A \beta)$ and intraneuronal aggregates of the microtubule-associated phosphoprotein tau. The purification of the respective aggregates (i.e., the amyloid plaques and the neurofibrillary tangles) from $A D$ brain tissue and the identification of their subunit proteins were followed by the discovery of disease-causing mutations in the cognate genes. In the case of the amyloid $\beta$-protein precursor (APP), missense mutations cause a form of early-onset, autosomal dominant $\mathrm{AD}$ that, although rare, has a neuropathological phenotype, including the presence of abundant neurofibrillary tangles, that is indistinguishable from that of the common, late-onset disorder (reviewed in ref. 1). Thus, the linkage of familial AD to APP mutations established the first discrete cause of the disorder. In the case of the tau protein, missense or splice mutations cause not $\mathrm{AD}$ but the

\footnotetext{
Address correspondence to: Dennis J. Selkoe, Center for Neurologic Diseases, Harvard Medical School, Brigham and Women's Hospital, 77 Avenue Louis Pasteur, Harvard Institutes of Medicine 730, Boston, Massachusetts 02115, USA. Phone: (617) 525-5200; Fax: (617) 525-5252; E-mail: selkoe@cnd.bwh.harvard.edu.

Conflict of interest: Dennis J. Selkoe is a consultant to Elan, PLC. Nonstandard abbreviations used: Alzheimer disease (AD); amyloid $\beta$-protein $(\mathrm{A} \beta)$; amyloid $\beta$-protein precursor (APP); transmembrane (TM); amyloid $\beta$-protein precursor intracellular domain (AICD); presenilin (PS); notch intracellular domain (NICD); insulin-degrading enzyme (IDE); cerebrospinal fluid (CSF); long-term potentiation (LTP).
}

less common disorder frontotemporal dementia with parkinsonism on chromosome 17 (FTDP-17) (reviewed in ref. 2). The latter disease is marked by severe neurofibrillary tangle formation, sufficient to lead to profound dementia and the demise of the host, but no aggregates of $A \beta$ develop. Therefore, genetics has largely resolved the contentious question of which of the two hallmark lesions, plaques or tangles, occurs first in $\mathrm{AD}$. It appears that $A \beta$ accumulation precedes and ultimately initiates the aggregation of wild-type tau protein in $\mathrm{AD}$, whereas the reverse sequence of pathogenesis has not been documented.

\section{The biogenesis of $A \beta$}

When APP was first cloned, the 40- and 42-residue A $\beta$ peptides were found to comprise the last 28 amino acids of its large, $\mathrm{N}$-terminal ectodomain plus the first 12 or 14 residues of its single transmembrane (TM) domain (Figure 1). This location predicted that the proteolytic cleavage creating the C-terminus of $\mathrm{A} \beta$ required some previous disruption of the membrane to allow access by the responsible protease (dubbed $\gamma$-secretase) into the phospholipid bilayer. It was therefore widely assumed that $A \beta$ production was not a primary event in $\mathrm{AD}$ but rather followed neuronal membrane injury. The subsequent discovery that both $A \beta 1-40$ and A $\beta 1-42$ peptides are constitutive products of cellular metabolism and occur in normal biological fluids throughout life disproved this notion (reviewed in ref. 3). The normal scission of APP near the middle of its TM domain to create $A \beta$ provided an initial example of the mechanism that came to be recognized as regulated intramembrane proteolysis (4). The unusual protease that effects this cleavage will be discussed below.

APP is a type 1 membrane glycoprotein that undergoes $\mathrm{N}$ - and O-linked glycosylation during its trafficking through the secretory pathway. At steady state, a small subset of APP molecules is found on the plasma membrane, and these can undergo ectodomain shedding by a proteolytic activity called $\alpha$-secretase (Figure 1). The disintegrin metalloproteinases ADAM 10 and ADAM 17 can serve as $\alpha$-secretases for $\operatorname{APP}(5,6)$. The 83-residue C-terminal fragment (C83) retained in the membrane can then undergo constitutive cleavage by $\gamma$-secretase to release the $\mathrm{p} 3$ peptide (residues 17-40/42 


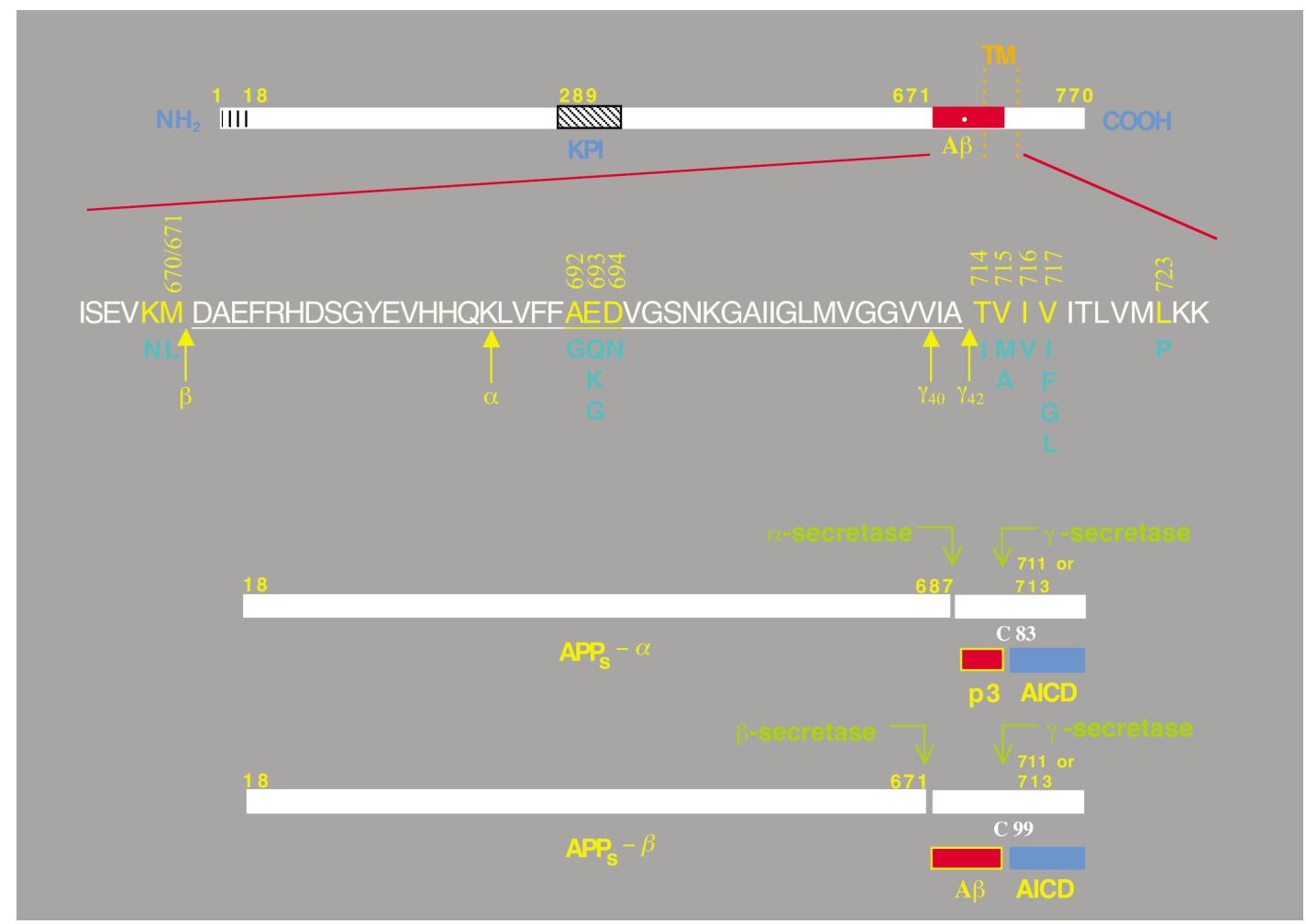

Figure 1

Schematic diagrams of the APP and its principal metabolic derivatives. The first line depicts the largest of the known APP alternate splice forms, comprising 770 amino acids. Regions of interest are indicated at their correct relative positions. A 17-residue signal peptide occurs at the $\mathrm{N}$-terminus (box with vertical lines). Two alternatively spliced exons of 56 and 19 amino acids are inserted at residue 289; the first contains a serine protease inhibitor domain of the Kunitz type (KPI). A single membrane-spanning domain (TM) at amino acids 700-723 is indicated by the vertical dotted lines. The $A \beta$ fragment includes 28 residues just outside the membrane plus the first 12-14 residues of the TM domain. In the second line, the sequence within APP that contains the A $\beta$ and TM regions is expanded. The underlined residues represent the A $\beta 1-42$ peptide. The blue letters below the wildtype sequence indicate the currently known missense mutations identified in certain patients with $A D$ and/or hereditary cerebral hemorrhage with amyloidosis. The three-digit numbers are codon numbers ( $\beta A P P 770$ isoform). In the third line, the arrow indicates the site (after residue 687) of a cleavage by $\alpha$-secretase that enables secretion of the large, soluble ectodomain of APP $\left(\operatorname{APP}_{S}-\alpha\right)$ into the medium and retention of the 83-residue $\mathrm{C}$-terminal fragment (C83) in the membrane. C83 can undergo cleavage by the protease called $\gamma$-secretase at residue 711 or residue 713 to release the $\mathrm{p} 3$ peptides. The fourth line depicts the alternative proteolytic cleavage after residue 671 by $\beta$-secretase that results in the secretion of the slightly truncated $\mathrm{APP}_{\mathrm{S}} \beta$ molecule and the retention of a 99 -residue C-terminal fragment. C99 can also undergo cleavage by $\gamma$-secretase to release the $A \beta$ peptides. Cleavage of both $\mathrm{C} 83$ and $\mathrm{C} 99$ by $\gamma$-secretase releases the AICD into the cytoplasm.

of $\mathrm{A} \beta$ ) plus the APP intracellular domain (AICD). The latter has proved exceedingly difficult to detect but was recently localized to both cytoplasm and nucleus (7-9). Alternatively, other APP holoproteins can be cleaved 16 residues $\mathrm{N}$-terminal to the $\alpha$-secretase site by a novel membrane-anchored aspartyl protease called $\beta$-secretase (or BACE1) (reviewed in ref. 10). This scission creates $C 99$, which is similarly processed by $\gamma$-secretase to yield A $\beta$ and AICD. Precisely where in the cell the $\beta$ and $\gamma$-secretases cleave APP is unsettled. Considerable evidence favors recycling endosomes, but the transGolgi network and secretory vesicles and perhaps even earlier secretory compartments (the endoplasmic reticulum and the cis-Golgi stacks) represent additional candidates (see, for example, ref. 11).

It should be borne in mind that the APP-processing events just summarized are entirely normal and occur to varying degrees in virtually all neural and non-neural cells throughout the body. Certain genetic defects that cause autosomal dominant $\mathrm{AD}$, such as mutations in APP or the presenilin (PS) genes PS1 and PS2, augment the amyloidogenic pathway of APP processing in all cells in a way that favors production of the highly self-aggregating $A \beta 1-42$ variant over the slightly shorter and less hydrophobic $A \beta 1-40$ form. $A \beta 1-42$ normally comprises only about $5-10 \%$ of total secreted $A \beta$ peptides, but this fraction rises to about $15-40 \%$ when either APP or PS is mutant.

\section{Presenilin: an AD gene product constitutes the active site of $\gamma$-secretase}

The recognition that familial AD is genetically heterogeneous was followed by the discovery of PS1 and PS2, mutations that may be responsible for roughly a third of the cases of early-onset ( $<65$ years), autosomal dominant $\mathrm{AD}$ (reviewed in ref. 12). More than 90 missense mutations identified to date in PS1 and at least six in PS2 cause the most aggressive forms of familial AD yet recognized, with some PS1 mutations producing symptoms of dementia as early as the twenties. Presenilins 
appear to have eight TM domains (13) and to be cleaved soon after their synthesis into two unequal fragments, which remain associated and form a heterodimer in vivo (Figure 2). The $\mathrm{AD}$-causing mutations are principally located within or adjacent to the TM segments, and they are presumed to distort the precise conformation of the molecule within the membrane. All AD-causing PS mutations expressed to date in cultured cells and/or transgenic mice increase the production of the $A \beta 42$ peptide. When such mice are crossed with mice expressing AD-causing mutations in human APP, the cerebral $\mathrm{A} \beta$ deposition that occurs in the latter mice is substantially accelerated (reviewed in ref. 14).

How do missense mutations in PS enhance the amyloidogenic processing of APP? The first clue came from deletion of the Ps1 gene in mice, which resulted in markedly altered skeletal and brain development in utero and perinatal mortality $(15,16)$. Embryonic neurons cultured from $P s 1^{-/-}$mice showed a striking $60-70 \%$ decrease in total $A \beta$ production, of both $A \beta 40$ and $A \beta 42$ (17). Also, a small fraction of APP molecules was found to coimmunoprecipitate with PS1 (18), suggesting that the two form transient complexes and that PS and APP localize in part to the same subcellular vesicles (19). Separate work using designed peptidomimetic inhibitors of $\gamma$-secretase suggested that this unknown enzyme had the properties of an aspartyl protease (20). Putting these and other observations together, our laboratory hypothesized that presenilin might actually be the $\gamma$-secretase. Close inspection of the sequence of presenilin revealed two conserved aspartate residues in adjacent TM domains (6 and 7) of all PS family members. Mutation of either aspartate in PS1 decreased cellular A $\beta$ secretion by approximately $60 \%$, akin to the effect of knocking out the entire PS1 gene (21). The coexpression of PS2 that also had one of its TM aspartates mutated reduced cellular $\mathrm{A} \beta$ secretion to undetectable levels (22).

We interpreted these results to suggest that the two aspartates constituted the active site of $\gamma$-secretase, an unprecedented intramembranous aspartyl protease that is activated by an autoproteolytic cleavage to create the biologically active heterodimeric form (21). This conclusion was met with substantial skepticism, given the lack of precedent for a polytopic aspartyl protease with its active site in the membrane. However, the model we proposed was reminiscent of the observation that the socalled site 2 protease (a metalloprotease implicated in cholesterol homeostasis that is responsible for cleaving the sterol regulatory element-binding protein [SREBP]) has its active site in the membrane (23). Strong support for the model came from the subsequent finding that $\gamma$-secretase inhibitors designed to mimic the transition state of a substrate with an aspartyl protease bind directly to presenilin heterodimers and no other cellular proteins $(24,25)$. Moreover, the region immediately around one of the two TM aspartates was found to be homologous to part of the presumed active site of a known bacterial aspartyl protease (26). Most recently, a related intramembrane protease, called signal peptide peptidase, has been discovered to have these two TM aspartate

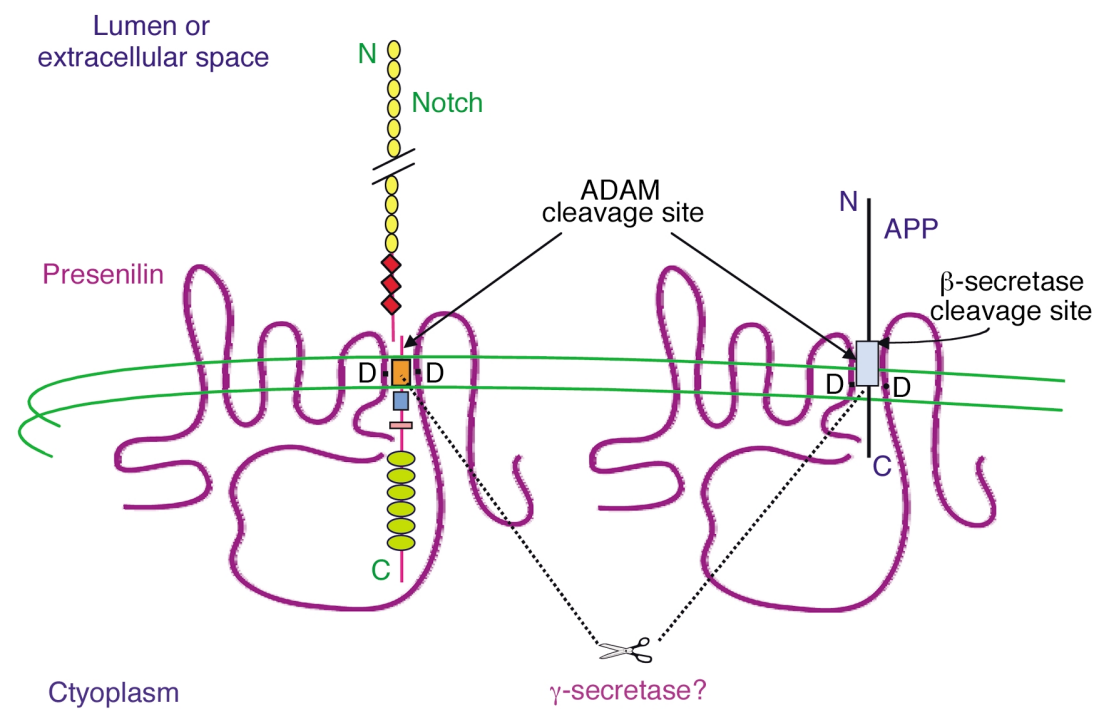

Figure 2

Hypothetical model of the role of PS in Notch and APP processing. The diagram shows the predicted eight-TM domain topology of PS, which occurs principally as a cleaved heterodimer. Some Notch and APP molecules form complexes with PS. Two aspartate residues (D) in TM6 and TM7 of PS are required for the cleavages of Notch and APP within their TM domains, and these are predicted to align with the respective sites of cleavage in the two substrates. It is likely that PS directly effects these cleavages, as part of a multi-protein complex (i.e., $\gamma$-secretase). PS-mediated proteolysis of both Notch and APP is preceded by ectodomain shedding mediated by certain ADAM proteases. Alternatively, a subset of APP holoproteins can undergo ectodomain shedding by $\beta$-secretase. Several motifs are depicted in Notch: EGF-like repeats (yellow circles), LNG repeats (red diamonds), a single TM (orange box), the RAM23 domain (blue square), a nuclear localization sequence (red rectangle), and six cdc10/ankyrin repeats (green ovals). Following the putative intramembranous cleavage mediated by PS, the Notch intracellular domain is released to the nucleus to activate transcription of target genes. APP contains the A $\beta$ region (light blue box), which is released into the lumen after sequential cleavages of APP by $\beta$-secretase and then $\gamma$-secretase/PS. Some of the AICD reaches the nucleus as well. 
motifs and to be capable of cleaving a substrate, thus providing very strong evidence that the presenilins are also intramembrane-cleaving aspartyl proteases (27).

Presenilin requires at least three other membrane proteins (nicastrin, Aph-1, and Pen-2) as cofactors to confer proteolytic activity (W.T. Kimberly and D.J. Selkoe, unpublished observations). Definitive confirmation of PS1 and PS2 as $\gamma$-secretases should now be possible if the four interacting proteins, assembled in vitro, can be shown to reconstitute APP-cleaving activity. However, the existing lines of evidence for this model already strongly support the amyloid hypothesis (more correctly, the $\mathrm{A} \beta$ hypothesis) of $\mathrm{AD}$ causation (3). Thus, all of the mutations identified in autosomal dominant forms of $\mathrm{AD}$ to date are found in either the substrate (APP) or the protease (PS) of the $\gamma$-secretase reaction. We speculate that each $\mathrm{AD}$-causing missense mutation in PS subtly alters the conformation of one or more of its TM domains to decrease the interaction of the two TM aspartates with the A $\beta 40-41$ peptide bond in the APP TM domain and/or increase the interaction with the $A \beta 42-43$ peptide bond, resulting in a relative overproduction of the highly self-aggregating A $\beta 1-42$ species throughout life.

\section{Presenilins are also $\gamma$-secretases for Notch and other integral membrane proteins}

As the work summarized above was proceeding, it became apparent that PS had another set of substrates, distinct from APP: the Notch family of cell-surface receptors (reviewed in ref. 28). Shortly after the human presenilins were cloned, the homologous gene product in Caenorbabditis elegans, sel-12, was shown to facilitate signaling by lin12 , the worm homologue of Notch (29). Notch is a major regulator of cell-fate determination in all metazoans. The binding of protein ligands, such as Delta or Jagged, to the Notch ectodomain had been shown to activate Notch signaling, initiating the transcription of nuclear genes that are transactivated by the Notch intracellular domain (NICD) (reviewed in ref. 30). How the cytoplasmic domain reached the nucleus was a matter of lively debate. Mounting evidence suggested that it did so as the result of two sequential proteolytic cleavages: first by ADAM 17 just 12 amino acids outside the TM domain (highly similar to the $\alpha$-secretase cleavage of APP) and then by a $\gamma$-secretase-like scission within the Notch TM domain $(31,32)$ (Figure 2). Loss-of-function mutations in the Drosophila presenilin gene produced a phenotype closely resembling that of Notch mutants and prevented the generation of the NICD fragment $(33,34)$. Furthermore, $\gamma$-secretase inhibitors lowered the proteolytic formation of NICD in a fashion indistinguishable from their effects on $A \beta(35)$. Similarly, deletion of both PS1 and PS2 in mammalian embryonic stem cells abolished both NICD production and $A \beta$ secretion $(36,37)$. The parallels between Notch and APP processing continue with the recent discovery that AICD participates in multiprotein complexes capable of driving gene transcription in the nucleus (38). Thus, the normal function of APP, like that of Notch, may be as a signaling receptor, although its ligands and downstream targets remain to be determined.
Accumulating evidence from numerous laboratories now supports the concept that the presenilins are key molecular switches that allow the release of cytoplasmic signaling domains from various single-TM polypeptides (39). Thus, work that began with a strict focus on the biology of $\mathrm{AD}$ has uncovered a unique proteolytic mechanism underlying what is likely to be a large number of signaling pathways. In this sense, one can postulate that $\mathrm{AD}$ arose in the human population because a highly conserved protein machine (the presenilin complex), which is crucial for developmental decisions in all multicellular organisms, has as one of its substrates a protein (APP), whose membranederived fragment can slowly aggregate and accumulate in the brain during postreproductive life. This accumulation occurs in virtually all older humans to some degree, and mutations in the substrate or the protease markedly accelerate the process, with devastating clinical consequences.

\section{A benign fate for cerebral $A \beta$ : degradation and clearance}

As with mutations that increase $A \beta$ production, factors that decrease its clearance or enhance its aggregation might well underlie some forms of the Alzheimer syndrome. An example appears to be provided by the ADpromoting effects of inheriting one or two E4 alleles of apoE. Humans expressing apoE4 proteins show no evidence of a general increase in $A \beta$ production; their plasma levels of $A \beta$ seem not to be elevated, and even the coexpression of APP with each of the human apoE isoforms does not affect cellular $A \beta$ generation. Instead, human neuropathological analyses (40), coupled with mouse modeling (41), suggest that inheritance of apoE4 leads to a rise in the steady-state levels of $A \beta$ in the brain, presumably by decreasing its clearance from the brain's extracellular space and/or enhancing its fibrillogenic potential. While the biochemical mechanism is not known, it is likely that apoE4-mediated rises in brain $A \beta$ levels represent a clearance failure.

Another regulator of $A \beta$ clearance is represented by the proteases in brain and non-neural tissues that are capable of degrading the peptide. This subject has only recently begun to receive experimental attention. While many purified proteases are capable of cleaving synthetic $A \beta$, only a few have so far been shown to do so under physiological conditions. Among these, neprilysin, a member of the neutral endopeptidase family of metalloproteases, is responsible for at least a portion of cerebral $A \beta$ degradation in vivo, as shown by the injection of inhibitors in rats (42) and the deletion of the gene in mice (43). Another candidate is insulindegrading enzyme (IDE), which can degrade synthetic $A \beta$ in vitro (44) but which has also emerged as a major $A \beta$-degrading protease in an unbiased screen performed on naturally secreted $A \beta$ in cell culture (45). The degree to which IDE mediates $A \beta$ degradation in vivo is currently under study. Interestingly, consideration of the $I D E$ gene on chromosome $10 \mathrm{q}$ as a candidate $\mathrm{AD}$ locus led to the discovery of linkage of nearby DNA markers to late-onset $\mathrm{AD}$ in the National Institute of Mental 
Health registry of $435 \mathrm{AD}$ families (46). However, other studies have obtained evidence of $A D$ linkage to $10 \mathrm{q}$ in a region considerably centromeric to $I D E$, so the identity of the putative $A D$ genes on $10 \mathrm{q}$ awaits resolution. Genes for other $A \beta$-degrading proteases, including members of the plasminogen pathway, are also under active study (reviewed in ref. 47).

Another potential clearance mechanism for $\mathrm{A} \beta$ aggregates is the phagocytosis of amyloid fibrils by cerebral microglia and their subsequent degradation by acid hydrolases in late endosomes and lysosomes. While a contribution by this route is possible, microaggregates of synthetic $A \beta 42$ shown to be internalized by cultured microglia and transported to lysosomes appear to be remarkably stable and to be degraded very slowly, leading to their severe accumulation inside cells (48). Therefore, it is unclear whether attempted $A \beta$ removal by this route is beneficial or rather induces a microglial-associated inflammatory response. Of related interest is the current debate as to whether the dramatic $A \beta$-clearing effects of active and passive $A \beta$ immunization in mice $(49,50)$ are due to the clearance of $A \beta$-antibody complexes by local microglia or to the transport of such complexes out of the brain into the cerebrospinal fluid (CSF) and blood (51).

\section{A malignant fate for cerebral $A \beta$ : aggregation}

If $A \beta$ peptides, particularly $A \beta 42$, are overproduced or insufficiently cleared, they become prone to aggregation into stable oligomers and larger polymers, apparently culminating in mature amyloid fibrils. In vitro aggregation studies of pure, synthetic $A \beta$ peptides of a single length indicate that the critical concentration that needs to be reached for aggregation to begin is relatively high, perhaps in the mid-micromolar range (52). In certain cell culture models, far lower (low nanomolar) levels of the heterogeneous $A \beta$ species, generated naturally by cells are associated with the appearance of small amounts of stable oligomers (53). This observation suggests that pro- (or anti-) aggregating cellular factors may regulate the conversion of monomers to dimers and higher oligomers. In vivo, it is assumed that high local concentrations of $A \beta$ sufficient to initiate and propagate oligomers are achieved in certain brain areas, eventually leading to the formation of microscopically visible deposits (plaques). Such high local levels have been demonstrated in the cortex of both $\mathrm{AD}$ patients and APP transgenic mice $(54,55)$. Many of the resultant in vivo deposits appear amorphous or diffuse, indicating that the $A \beta$ occurs principally in nonfibrillar (fine granular) aggregates. Whether some of this "pre-amyloid" material is similar to the protofibrils that form as metastable intermediates during synthetic $A \beta$ fibrillization in vitro $(56,57)$ remains to be determined.

A central question about the $\mathrm{A} \beta$ hypothesis concerns which assembly forms of the peptide - monomers, oligomers (e.g., protofibrils), and/or mature amyloid fibrils - may be neurotoxic. Although synthetic $A \beta$ fibrils reproducibly induce neuronal injury and loss in cell culture and after intracortical injection (58), the aggregates employed are usually complex mixtures of assembly forms that are difficult to define and quantify individually. The occurrence of neuritic dystrophy, microglial activation, and astrocytosis within amyloid plaques has long supported the hypothesis that these mature, fibrillar lesions can induce injury. However, the fibrils are likely to be in equilibrium with abundant oligomers and monomers in their immediate vicinity, since amyloid fibrils have both an on-rate and an off-rate. In postmortem AD brain tissue and the brains of older APP transgenic mice, the complex mixture of $A \beta$ assembly forms precludes assignment of toxic effects to a particular species. However, young APP transgenic mice show altered synaptic morphology and electrophysiological changes well before the microscopic appearance of $A \beta$ deposits $(59,60)$. Because such mice show steadily rising total $A \beta$ levels in the brain before plaques develop (55), $A \beta$ monomers and/or oligomers could be responsible.

In an attempt to discern which species of $A \beta$ may be synaptotoxic in vivo, we have recently taken advantage of the production by certain cultured cells of low levels $(<1 \mathrm{nM})$ of highly stable oligomers (dimers, trimers, and tetramers) of naturally secreted human $A \beta$ (53). Microinjection of the conditioned media into the lateral ventricle of anesthetized rats potently blocked the maintenance of hippocampal long-term potentiation (LTP), an electrophysiological correlate of synaptic plasticity (61). This medium is devoid of $A \beta$ fibrils or protofibrils. Immunodepletion of all $A \beta$ species fully reversed the block of LTP. To separate the effects of monomers and oligomers, we used the ability of IDE to quantitatively degrade $A \beta$ monomers while leaving the oligomers unaltered (45). When IDE-treated medium devoid of monomers was microinjected, LTP was still blocked, enabling us to attribute the inhibition to the oligomers. This was confirmed by treating the same cells with doses of a $\gamma$-secretase inhibitor sufficient to lower oligomer levels to undetectable levels while preserving a substantial fraction $(-60 \%)$ of the monomer. Microinjection of this medium allowed normal LTP. Therefore, natural oligomers of human $A \beta$, in the absence of monomers and amyloid fibrils, can disrupt synaptic plasticity in vivo at concentrations found in human brain and CSF. It remains to be seen whether such a phenomenon occurs in the $\mathrm{AD}$ hippocampus and could result in synaptic dysfunction - and thus memory impairment prior to widespread neuronal death.

While these and other data suggest a direct action of $A \beta$ oligomers on synapses, $A \beta$ aggregates could also lead indirectly to neurotoxicity by activating microglia and astrocytes and inducing a local inflammatory response, including the triggering of the complement cascade (reviewed in ref. 62). It is not yet clear whether microglia are the earliest cellular responders to the accumulation of $A \beta$ or whether neurons react just as quickly. If microglia represent the first cellular target, some of the subsequent synaptic and dendritic changes in $\mathrm{AD}$ cortex may be caused by inflammatory and neurotoxic factors released by microglia. During the long presymptomatic phase of $\mathrm{AD}$, it is likely that many molecular and cellular changes develop contempora- 
neously. As a result, attempts to identify one or a few "master" pathways of AD-type neurotoxicity and inhibit them pharmacologically may well prove elusive. Given the difficulty of ascertaining the precise sequence of biochemical changes, even in mouse models of the disease, neuronal and glial toxicity, per se, are less attractive therapeutic targets than is lowering the levels of $A \beta$ monomers and oligomers.

\section{The $A \beta$ cascade of AD}

While controversy still swirls around the concept that $\mathrm{AD}$ is fundamentally a cerebral amyloidosis, the number of genetic, cell biological, neuropathological, and animal modeling studies that support an initiating role for $\mathrm{A} \beta$ is impressive and growing apace. Because the phenotypes of the known genetic forms of $\mathrm{AD}$ are essentially indistinguishable from that of common, late-onset disease, all genetic (and environmental) factors ultimately found to predispose to $\mathrm{AD}$ may do so by altering the production or clearance of $A \beta$ or heightening the cytopathological responses to $A \beta$ aggregates. As discussed above, oligomeric intermediates of $A \beta$, rather than mature amyloid fibrils, may turn out to be the principal form though which the peptide exerts its ill effects. Therefore, the "amyloid cascade" of AD is gradually being refined into a related "A $\beta$ cascade." $A \beta$ oligomers may exert complex effects on surrounding neurons, microglia, and astrocytes, the cumulative effect of which is to subtly alter synaptic function, and thus information storage and retrieval. The tau-containing dystrophic neurites and neurofibrillary tangles that develop in "thinking" parts of the brain in $\mathrm{AD}$ are likely to be a consequence of $\mathrm{A} \beta$ build-up; this sequence is supported by recent studies of mice bearing both human $A \beta$ and human tau $(63,64)$. While the loss of tau function represented by paired helical filaments(PHF) formation in $\mathrm{AD}$ is no doubt deleterious, there are likely to be parallel molecular changes not dependent on tau that also contribute to neuronal dysfunction. The inexorable outcome of this complex process is the interruption of neurotransmission, which the physician currently attempts to correct with cholinergic replacement therapy (i.e., acetylcholinesterase inhibitors), usually to little avail.

Although the outlines of a pathogenic cascade that could explain cognitive dysfunction in Alzheimer patients are emerging, many important questions remain. What are the relative contributions of extracellular and intraneuronal $A \beta$ accumulation in the initiation of neurotoxicity? Can one confirm that smaller $A \beta$ assemblies (stable but diffusible oligomers) are the principal neuron-injuring and microglia-activating species? Is apoptosis of neurons an important part of the pathogenic cascade that, if inhibited, would slow or prevent brain dysfunction? Perhaps most challenging is how to explain the remarkably selective vulnerability of neuronal populations to the disease. Local and regional differences in the pathogenic process seem to arise on at least two broad levels. First, $A \beta 42$ can accumulate chronically in some brain regions (e.g., cerebellum, striatum, and thalamus) with very little evolution to fibril-rich amyloid plaques and little associated neu- ritic and glial pathology. This suggests the existence of pro- or antiaggregating factors that vary among brain regions and promote or prevent $\mathrm{A} \beta 42$ aggregation into toxic oligomeric forms. Second, even in regions (e.g., hippocampus and cerebral cortex) where abundant $A \beta$ oligomerization and fibrillization occur, some neurons and their processes appear to undergo no cytotoxic response. This suggests an intrinsic ability of certain neurons to resist $A \beta$-mediated cytotoxicity.

\section{A new diagnostic and treatment paradigm emerges}

Although questions abound, the field may have accrued enough information about pathogenesis to envision a new way to manage, and ultimately prevent, the disease. In the future, individuals reaching their fifties or beyond may be offered a specific risk-assessment profile to determine their likelihood of developing AD. Such an assessment, modeled on that now widely used to judge the risk of serious atherosclerotic disease, would include inquiry about a positive family history of $\mathrm{AD}$ or a related dementia, identification of specific predisposing genetic factors, structural and functional brain imaging to detect evidence of presymptomatic lesions (including specific "A $\beta$ scans"), and measurement of $A \beta 42$, tau, and other markers of the neuropathology in CSF and, in the case of $A \beta$, in plasma. Based on further epidemiological experience with such assessment measures, it would be possible to estimate - first crudely and later more accurately - the likelihood that an individual will develop AD. Those at appreciable risk can be offered preventive treatment with one of several agents that are just now beginning to enter clinical trials. These include small-molecule inhibitors of $\gamma$-secretase (one of which has been used in a phase 1 study) or $\beta$-secretase (in preclinical development), as well as active or passive "A $\beta$ vaccination". The last approach derives from mouse studies in which the repetitive parenteral administration of synthetic $A \beta$ peptide was found to induce an antibody response that lowered cerebral $A \beta$ levels (49). Precisely how anti-A $\beta$ antibodies do this is under study. Microglial clearance of $A \beta$-antibody complexes (50) and/or an antibody-mediated redistribution of soluble $A \beta$ from brain into CSF and then plasma (51) may be involved. Mucosal A $\beta$ vaccines might also be effective (65).

If these or other approaches based on the $\mathrm{A} \beta$ hypothesis are shown to slow the progression of cognitive impairment, then $\mathrm{AD}$ will emerge as a prime example of the power of reductionist science to ameliorate a disease of the most complex of biological systems, the human cerebral cortex.

1. Goate, A.M. 1998. Monogenetic determinants of Alzheimer's disease: APP mutations. Cell. Mol. Life Sci. 54:897-901.

2. Goedert, M., and Spillantini, M.G. 2000. Tau mutations in frontotemporal dementia FTDP-17 and their relevance for Alzheimer's disease. Biochim. Biophys. Acta. 1502:110-121.

3. Selkoe, D.J. 2001. Alzheimer's disease: genes, proteins and therapies. Physiol. Rev. 81:742-761.

4. Brown, M.S., Ye, J., Rawson, R.B., and Goldstein, J.L. 2000. Regulated intramembrane proteolysis: a control mechanism conserved from bacteria to humans. Cell. 100:391-398.

5. Buxbaum, J.D., et al. 1998. Evidence that tumor necrosis factor alpha converting enzyme is involved in regulated alpha-secretase cleavage of the Alzheimer amyloid protein precursor. J. Biol. Chem. 273:27765-27767.

6. Kojro, E., Gimpl, G., Lammich, S., Marz, W., and Fahrenholz, F. 2001. Low 
cholesterol stimulates the nonamyloidogenic pathway by its effect on the alpha-secretase ADAM 10. Proc. Natl. Acad. Sci. USA. 98:5815-5820.

7. Kimberly, W.T., Zheng, J.B., Guenette, S.Y., and Selkoe, D.J. 2001. The intracellular domain of the beta-amyloid precursor protein is stabilized by Fe65 and translocates to the nucleus in a notch-like manner. J. Biol. Chem. 276:40288-40292.

8. Sastre, M., et al. 2001. Presenilin-dependent gamma-secretase processing of beta-amyloid precursor protein at a site corresponding to the S3 cleavage of Notch. EMBO Rep. 2:835-841.

9. Cupers, P., Orlans, I., Craessaerts, K., Annaert, W., and De Strooper, B. 2001. The amyloid precursor protein (APP)-cytoplasmic fragment generated by gamma-secretase is rapidly degraded but distributes partially in a nuclear fraction of neurones in culture. J. Neurochem. 78:1168-1178.

10. Vassar, R., and Citron, M. 2000. Abeta-generating enzymes: recent advances in beta- and gamma-secretase research. Neuron. 27:419-422.

11. Wilson, C.A., Doms, R.W., Zheng, H., and Lee, V.M. 2002. Presenilins are not required for Abeta42 production in the early secretory pathway. Nat. Neurosci. 5:849-855.

12. St George-Hyslop, P.H. 2000. Molecular genetics of Alzheimer's disease. Biol. Psychiatry. 47:183-199.

13. Li, X., and Greenwald, I. 1998. Additional evidence for an eight-transmembrane-domain topology for Caenorhabditis elegans and human presenilins. Proc. Natl. Acad. Sci. USA. 95:7109-7114.

14. Price, D.L., et al. 2000. The value of transgenic models for the study of neurodegenerative diseases. Ann. NY Acad. Sci. 920:179-191.

15. Shen, J., et al. 1997. Skeletal and CNS defects in presnilin-1 deficient mice. Cell. 89:629-639.

16. Wong, P., et al. 1997. Presenilin 1 is required for Notch 1 and D111 expression in the paraxial mesoderm. Nature. 397:288-292.

17. De Strooper, B., et al. 1998. Deficiency of presenilin-1 inhibits the normal cleavage of amyloid precursor protein. Nature. 391:387-390.

18. Xia, W., Zhang, J., Perez, R., Koo, E.H., and Selkoe, D.J. 1997. Interaction between amyloid precursor protein and presenilins in mammalian cells: implications for the pathogenesis of Alzheimer's disease. Proc. Natl. Acad. Sci.USA. 94:8208-8213.

19. Zhang, J., et al. 1998. Subcellular distribution and turnover of presenilins in transfected cells. J. Biol. Chem. 273:12436-12442.

20. Wolfe, M.S., et al. 1998. A substrate-based difluoro ketone selectively inhibits Alzheimer's gamma-secretase activity. J. Med. Chem. 41:6-9.

21. Wolfe, M.S., et al. 1999. Two transmembrane aspartates in presenilin-1 required for presenilin endoproteolysis and $\gamma$-secretase activity. Nature. 398:513-517.

22. Kimberly, W.T., Xia, W., Rahmati, R., Wolfe, M.S., and Selkoe, D.J. 2000. The transmembrane aspartates in presenilin 1 and 2 are obligatory for $\gamma$-secretase activity and amyloid $\beta$-protein generation. J. Biol. Chem. 275:3173-3178.

23. Rawson, R.B., et al. 1997. Complementation cloning of S2P, a gene encoding a putative metalloprotease required for intramembrane cleavage of SREBPs. Mol. Cell. 1:47-57.

24. Esler, W.P., et al. 2000. Transition-state analogue inhibitors of $\gamma$-secretase bind directly to presenilin-1. Nat. Cell Biol. 2:428-434.

25. Li, Y.-M., et al. 2000. Photoactivated $\gamma$-secretase inhibitors directed to the active site covalently label presenilin 1. Nature. 405:689-694.

26. Steiner, H., et al. 2000. Glycine 384 is required for presenilin-1 function and is conserved in bacterial polytopic aspartyl proteases. Nat. Cell Biol. 2:848-851.

27. Weihofen, A., Binns, K., Lemberg, M.K., Ashman, K., and Martoglio, B. 2002. Identification of signal peptide peptidase, a presenilin-type aspartic protease. Science. 296:2215-2218.

28. Haass, C., and De Strooper, B. 1999. The presenilins in Alzheimer's disease: proteolysis holds the key. Science. 286:916-919.

29. Levitan, D., and Greenwald, I. 1995. Facilitation of lin-12-mediated signalling by sel-12, a Caenorbabditis elegans S182 Alzheimer's disease gene. Nature. 377:351-354.

30. Artavanis-Tsakonas, S., Rand, M.D., and Lake, R.J. 1999. Notch signaling: cell fate control and signal integration in development. Science. 284:770-776.

31. Schroeter, E.H., Kisslinger, J.A., and Kopan, R. 1998. Notch-1 signalling requires ligand-induced proteolytic release of intracellular domain. Nature. 393:382-386.

32. Logeat, F., et al. 1998. The Notch1 receptor is cleaved constitutively by a furin-like convertase. Proc. Natl. Acad. Sci. USA. 95:8108-8112.

33. Struhl, G., and Greenwald, I. 1999. Presenilin is required for activity and nuclear access of Notch in Drosophila. Nature. 398:522-525.

34. Ye, Y., Lukinova, N., and Fortini, M.E. 1999. Neurogenic phenotypes and altered Notch processing in Drosophila Presenilin mutants. Nature. 398:525-529.

35. De Strooper, B., et al. 1999. A presenilin-1-dependent gamma-secretaselike protease mediates release of Notch intracellular domain. Nature. 398:518-522.
36. Song, W., et al. 1999. Proteolytic release and nuclear translocation of Notch- 1 are induced by presenilin- 1 and impaired by pathogenic presenilin-1 mutations. Proc. Natl. Acad. Sci. USA. 96:6959-6963.

37. Herreman, A., et al. 2000. Total inactivation of gamma-secretase activity in presenilin-deficient embryonic stem cells. Nat. Cell Biol. 2:461-462.

38. Cao, X., and Sudhof, T.C. 2001. A transcriptionally [correction of transcriptively] active complex of APP with Fe65 and histone acetyltransferase Tip60. Science. 293:115-120.

39. Struhl, G., and Adachi, A. 2000. Requirements for presenilin-dependent cleavage of notch and other transmembrane proteins. Mol. Cell. 6:625-636.

40. Schmechel, D.E., et al. 1993. Increased amyloid $\beta$-peptide deposition in cerebral cortex as a consequence of apolipoprotein E genotype in lateonset Alzheimer disease. Proc. Natl. Acad. Sci. USA. 90:9649-9653.

41. Holtzman, D.M., et al. 2000. Apolipoprotein E isoform-dependent amyloid deposition and neuritic degeneration in a mouse model of Alzheimer's disease. Proc. Natl. Acad. Sci. USA. 97:2892-2897.

42. Iwata, N., et al. 2000. Identification of the major Abeta1-42-degrading catabolic pathway in brain parenchyma: suppression leads to biochemical and pathological deposition. Nat. Med. 6:143-150.

43. Iwata, N., et al. 2001. Metabolic regulation of brain Abeta by neprilysin. Science. 292:1550-1552

44. Kurochkin, I.V., and Goto, S. 1994. Alzheimer's $\beta$-amyloid peptide specifically interacts with and is degraded by insulin degrading enzyme. FEBS Lett. 345:33-37.

45. Qiu, W.Q., et al. 1998. Insulin degrading enzyme regulates the level of monomeric amyloid $\beta$-protein extracellularly via degradation and oligomeriztion. J. Biol. Chem. 273:32730-32738.

46. Bertram, L., et al. 2000. Evidence for genetic linkage of Alzheimer's disease to chromosome 10q. Science. 290:2302-2303.

47. Selkoe, D. 2001. Clearing the brain's amyloid cobwebs. Neuron. 32:177-180.

48. Paresce, D.M., Chung, H., and Maxfield, F.R. 1997. Slow degradation of aggregates of the Alzheimer's disease amyloid $\beta$-protein by microglial cells. J. Biol. Chem. 272:29390-29397.

49. Schenk, D., et al. 1999. Immunization with amyloid- $\beta$ attenuates Alzheimerdisease-like pathology in the PDAPP mouse. Nature. 400:173-177.

50. Bard, F., et al. 2000. Peripherally administered antibodies against amyloid beta-peptide enter the central nervous system and reduce pathology in a mouse model of Alzheimer disease. Nat. Med. 6:916-919.

51. DeMattos, R.B., et al. 2001. Peripheral anti-A beta antibody alters CNS and plasma A beta clearance and decreases brain A beta burden in a mouse model of Alzheimer's disease. Proc. Natl. Acad. Sci. USA. 98:8850-8855.

52. Lomakin, A., Chung, D.S., Benedek, G.B., Kirschner, D.A., and Teplow, D.A. 1996. On the nucleation and growth of amyloid $\beta$-protein fibrils: detection of nuclei and quantitation of rate constants. Proc. Natl. Acad. Sci. USA. 93:1125-1129.

53. Walsh, D.M., Tseng, B.P., Rydel, R.E., Podlisny, M.B., and Selkoe, D.J. 2000 . Detection of intracellular oligomers of amyloid $\beta$-protein in cells derived from human brain. Biochemistry. 39:10831-10839.

54. Gravina, S.A., et al. 1995. Amyloid $\beta$ protein $(A \beta)$ in Alzheimer's disease brain. J. Biol. Chem. 270:7013-7016.

55. Johnson-Wood, K., et al. 1997. Amyloid precursor protein processing and A $\beta 42$ deposition in a transgenic mouse model of Alzheimer disease. Proc. Natl. Acad. Sci. USA. 94:1550-1555.

56. Harper, J.D., Wong, S.S., Lieber, C.M., and Lansbury, P.T., Jr. 1997. Observation of metastable $A \beta$ amyloid protofibrils by atomic force microscopy. Chem. Biol. 4:119-125.

57. Walsh, D.M., et al. 1997. Amyloid $\beta$-protein fibrillogenesis: detection of a protofibrillar intermediate. J. Biol. Chem. 272:22364-22374.

58. Yankner, B.A. 1996. Mechanisms of neuronal degeneration in Alzheimer's disease. Neuron. 16:921-932.

59. Hsia, A.Y., et al. 1999. Plaque-independent disruption of neural circuits in Alzheimer's disease mouse models. Proc. Natl. Acad. Sci. USA. 96:3228-3233.

60. Mucke, L., et al. 2000. High-level neuronal expression of abeta 1-42 in wild-type human amyloid protein precursor transgenic mice: synaptotoxicity without plaque formation. J. Neurosci. 20:4050-4058.

61. Walsh, D., et al. 2002. Naturally secreted oligomers of the Alzheimer amyloid $\beta$-protein potently inhibit hippocampal long-term potentiation in vivo. Nature. 416:535-539.

62. Rogers, J., et al. 1996. Inflammation and Alzheimer's disease pathogenesis. Neurobiol. Aging. 17:681-686.

63. Lewis, J., et al. 2001. Enhanced neurofibrillary degeneration in transgenic mice expressing mutant tau and APP. Science. 293:1487-1491.

64. Gotz, J., Chen, F., van Dorpe, J., and Nitsch, R.M. 2001. Formation of neurofibrillary tangles in P301l tau transgenic mice induced by Abeta 42 fibrils. Science. 293:1491-1495.

65. Weiner, H.L., et al. 2000. Nasal administration of amyloid-beta peptide decreases cerebral amyloid burden in a mouse model of Alzheimer's disease. Ann. Neurol. 48:567-579. 